University of Shizuoka

US-01-02, v3

June 2001

hep-ph/0104226

\title{
Can the Zee Model Explain the Observed Neutrino Data?
}

\author{
Yoshio Koidef \\ Department of Physics, University of Shizuoka \\ 52-1 Yada, Shizuoka 422-8526, Japan
}

\begin{abstract}
The eigenvalues and mixing angles in the Zee model are investigated parameterindependently. When we require $\left|\Delta m_{12}^{2} / \Delta m_{23}^{2}\right| \ll 1$ in order to understand the solar and atmospheric data simultaneously, the only solution is one which gives bi-maximal mixing. It is pointed out that the present best-fit value of $\sin ^{2} 2 \theta_{\text {solar }}$ in the MSW LMA solution cannot be explained within the framework of the Zee model, because we derive a severe constraint on the value of $\sin ^{2} 2 \theta_{\text {solar }}, \sin ^{2} 2 \theta_{\text {solar }} \geq 1-(1 / 16)\left(\Delta m_{\text {solar }}^{2} / \Delta m_{\text {atm }}^{2}\right)^{2}$.
\end{abstract}

PACS numbers: $14.60 . \mathrm{Pq}$

* E-mail: koide@u-shizuoka-ken.ac.jp 
Of the neutrino mass matrix models proposed currently, the Zee model[1] is a very attractive one, because the model can naturally leads to a large neutrino mixing with few parameters [2, 3, 团. The neutrino mass matrix $M_{\nu}$ in the basis on which the charged lepton mass matrix $M_{e}$ is diagonal is given by the form

$$
M_{\nu} \simeq m_{0}\left(\begin{array}{ccc}
0 & a & c \\
a & 0 & b \\
c & b & 0
\end{array}\right)
$$

where

$$
\begin{aligned}
& a=f_{e \mu}\left(m_{\mu}^{2}-m_{e}^{2}\right), \\
& b=f_{\mu \tau}\left(m_{\tau}^{2}-m_{\mu}^{2}\right), \\
& c=f_{\tau e}\left(m_{e}^{2}-m_{\tau}^{2}\right),
\end{aligned}
$$

and $f_{e \mu}, f_{\mu \tau}$ and $f_{\tau e}$ are lepton-number violating Yukawa coupling constants with the Zee scalar $h^{+}$. It is known that if we consider a Zee mass matrix with $a=c \gg|b|$, the model

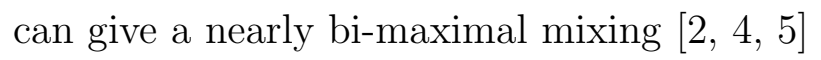

$$
U_{\nu}=\left(\begin{array}{ccc}
\cos \theta & -\sin \theta & 0 \\
\frac{1}{\sqrt{2}} \sin \theta & \frac{1}{\sqrt{2}} \cos \theta & -\frac{1}{\sqrt{2}} \\
\frac{1}{\sqrt{2}} \sin \theta & \frac{1}{\sqrt{2}} \cos \theta & \frac{1}{\sqrt{2}}
\end{array}\right)
$$

where

$$
\begin{gathered}
\tan \theta=\sqrt{-m_{\nu 1} / m_{\nu 2}}, \\
\Delta m_{12}^{2}=m_{\nu 1}^{2}-m_{\nu 2}^{2} \simeq 2 \sqrt{2} a b, \quad \Delta m_{23}^{2}=m_{\nu 2}^{2}-m_{\nu 3}^{2} \simeq 2 a^{2},
\end{gathered}
$$

which lead to

$$
\frac{\Delta m_{12}^{2}}{\Delta m_{23}^{2}} \simeq \sqrt{2} \frac{b}{a} .
$$

Furthermore, if we assume a badly broken horizontal symmetry $\mathrm{SU}(3)_{H}$ and we put a simple ansatz on the transition matrix elements in the infinite momentum frame (not on the mass matrix), we can obtain the relations [6]

$$
f_{i j}=\varepsilon_{i j k} \frac{m_{k}^{e}}{m_{i}^{e}+m_{j}^{e}} f,
$$


where $f$ is a common factor and $m_{i}^{e}=\left(m_{e}, m_{\mu}, m_{\tau}\right)$, so that we can predict

$$
\frac{\Delta m_{12}^{2}}{\Delta m_{23}^{2}} \simeq \sqrt{2} \frac{m_{e}}{m_{\mu}}=6.7 \times 10^{-3}
$$

which is in excellent agreement with the observed value (best fit value) [7, 8]

$$
\left(\frac{\Delta m_{\text {solar }}^{2}}{\Delta m_{\text {atm }}^{2}}\right)_{\text {exp }} \simeq \frac{2.2 \times 10^{-5} \mathrm{eV}^{2}}{3.2 \times 10^{-3} \mathrm{eV}^{2}}=6.9 \times 10^{-3}
$$

Thus, the Zee model is very attractive from the phenomenological point of view. However, most authors who investigated the Zee neutrino mass matrix have failed to give the observed value $\sin ^{2} 2 \theta_{\text {solar }} \simeq 0.7$ in the MSW LMA solution [9], although it is easy to give the bi-maximal mixing (3). It is a serious problem for the Zee model whether the model can fit the observed value $\sin ^{2} 2 \theta_{\text {solar }} \simeq 0.7$ or not. In the present paper, from a parameter-independent study of the Zee neutrino mass matrix (1), we conclude that the value of $\sin ^{2} 2 \theta_{\text {solar }}$ must satisfy a severe constraint $\sin ^{2} 2 \theta_{\text {solar }} \geq 1-$ $(1 / 16)\left(\Delta m_{\text {solar }}^{2} / \Delta m_{\text {atm }}^{2}\right)^{2}$ in the Zee model with $\Delta m_{\text {solar }}^{2} / \Delta m_{\text {atm }}^{2} \ll 1$. The similar subject has also been discussed by Frampton and Glashow [10]. However, the constraint obtained in the present paper is more explicit and very severe. This constraint will force us to abandon the Zee model or to modify the original Zee model to an extended version with some additional terms.

The mass matrix (1) is diagonalized by a unitary matrix $U_{\nu}$ as

$$
U_{\nu}^{T} M_{\nu} U_{\nu}=D_{\nu} \equiv \operatorname{diag}\left(m_{1}, m_{2}, m_{3}\right) .
$$

The Maki-Nakagawa-Sakata (MNS) [11] matrix $U_{M N S}$ is given by $U_{M N S}=U_{\nu}$, because the charged lepton mass matrix is diagonal in the Zee model. In order to obtain the relations among the mass matrix parameters and the mass eigenvalues, we define the Hermitian matrix $H_{\nu}$ as

$$
H_{\nu}=M_{\nu}^{\dagger} M_{\nu}
$$

so that we obtain

$$
U_{\nu}^{\dagger} H_{\nu} U_{\nu}=D_{\nu}^{*} D_{\nu}=\operatorname{diag}\left(\left|m_{1}\right|^{2},\left|m_{2}\right|^{2},\left|m_{3}\right|^{2}\right)
$$

The form of $H_{\nu}$ is explicitly given by

$$
H_{\nu}=H_{0}-H_{1}
$$

where

$$
H_{0}=m_{0}^{2}\left(|a|^{2}+|b|^{2}+|c|^{2}\right) \mathbf{1},
$$




$$
H_{1}=m_{0}^{2}\left(\begin{array}{ccc}
|b|^{2} & -c^{*} b & -a^{*} b \\
-b^{*} c & |c|^{2} & -a^{*} c \\
-b^{*} a & -c^{*} a & |a|^{2}
\end{array}\right)
$$

and 1 is a $3 \times 3$ unit matrix. The matrix $H_{1}$ is diagonalized as

$$
U_{\nu}^{\dagger} H_{1} U_{\nu}=\operatorname{diag}\left(h_{1}, h_{2}, h_{3}\right)
$$

and the eigenvalues $h_{i}$ satisfy the equation

$$
h_{i}^{3}-\left(|a|^{2}+|b|^{2}+|c|^{2}\right) m_{0}^{2} h_{i}^{2}+4|a|^{2}|b|^{2}|c|^{2} m_{0}^{6}=0
$$

By re-defining $m_{0}$, without losing generality, we can take $|a|^{2}+|b|^{2}+|c|^{2}=1$, so that the solutions $h_{i}=m_{0}^{2} x_{i}$ are described only by one parameter

$$
|q|^{2}=|a|^{2}|b|^{2}|c|^{2}
$$

as

$$
x_{i}^{3}-x_{i}^{2}+4|q|^{2}=0 .
$$

The equation (19) has three real solutions $x_{i}$ only when $|q|^{2}<1 / 27$. The behaviors of the solutions $x_{i}$ are illustrated in Fig. 1. The mass squared $\left|m_{i}\right|^{2}$ is given by

$$
\left|m_{i}\right|^{2}=\left(1-x_{i}\right) m_{0}^{2}
$$

From Fig. 1, we find that the cases which can explain the observed fact $\left|\Delta m_{12}^{2} / \Delta m_{23}^{2}\right| \ll 1$ are only the cases with $|q|^{2} \simeq 1 / 27$ and $|q|^{2} \simeq 0$.

For the case with $|q|^{2} \simeq 1 / 27$, by putting

$$
x_{1}=\frac{2}{3}+\varepsilon_{1}, \quad x_{2}=\frac{2}{3}-\varepsilon_{2}, \quad x_{3}=-\frac{1}{3}+\varepsilon_{3}, \quad|q|^{2}=\frac{1}{27}-\varepsilon_{q}^{2},
$$

and by putting (21) into the equation (19), we can obtain

$$
\varepsilon_{1} \simeq \varepsilon_{2} \simeq 2 \varepsilon_{q}, \quad \varepsilon_{3} \simeq 4 \varepsilon_{q}^{2}
$$

so that we obtain

$$
\frac{\Delta m_{21}^{2}}{\Delta m_{32}^{2}} \simeq 4 \varepsilon_{q} .
$$

On the other hand, from Eq. (16), we obtain

$$
\left(H_{1} / m_{0}^{2}\right)_{i i}=\left|U_{\nu i 1}\right|^{2} x_{1}+\left|U_{\nu i 2}\right|^{2} x_{2}+\left|U_{\nu i 3}\right|^{2} x_{3}
$$




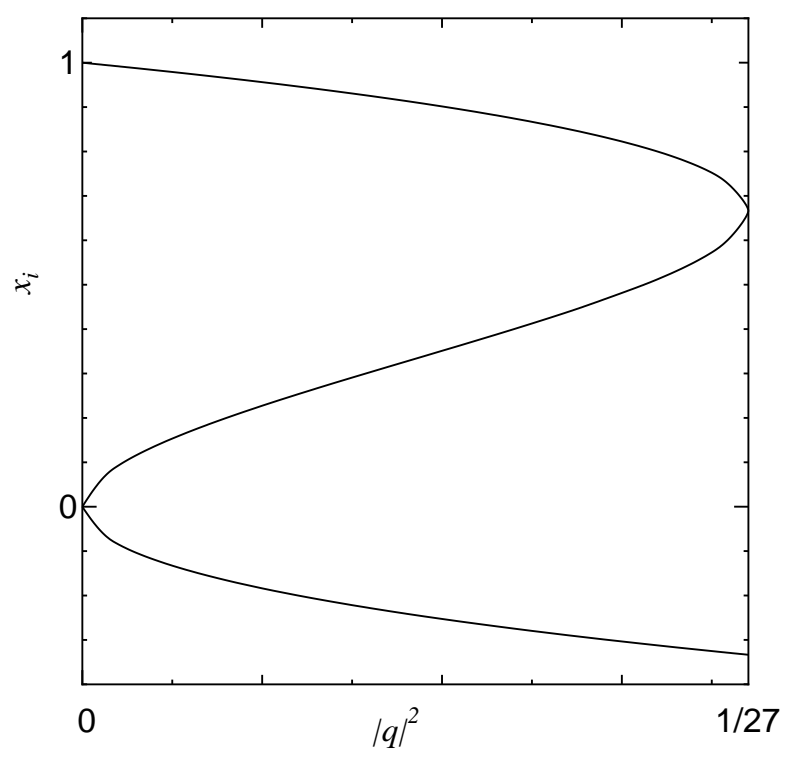

Figure 1: The eigenvalues $x_{i}(i=1,2,3)$ versus $|q|^{2}$. The solutions $x_{i}$ of the equation (19) have real three values only in the range $0 \leq|q|^{2} \leq 1 / 27$. The values $x_{i}$ take $(0,0,1)$ and $(-1 / 3,2 / 3,2 / 3)$ at $|q|^{2}=0$ and $|q|^{2}=1 / 27$, respectively. The mass eigenvalues $\left|m_{i}\right|^{2}$ are given by $\left|m_{i}\right|^{2}=\left(1-x_{i}\right) m_{0}^{2}$.

For the case with $|q|^{2} \simeq 1 / 27$, Eq. (24) gives

$$
\left(H_{1} / m_{0}^{2}\right)_{i i} \simeq \frac{2}{3}-\left|U_{\nu i 3}\right|^{2}+2 \varepsilon_{q}\left(\left|U_{\nu i 1}\right|^{2}-\left|U_{\nu i 2}\right|^{2}\right)
$$

i.e.,

$$
|b|^{2} \simeq \frac{2}{3}-\left|U_{\nu 13}\right|^{2}, \quad|c|^{2} \simeq \frac{2}{3}-\left|U_{\nu 23}\right|^{2}, \quad|a|^{2} \simeq \frac{2}{3}-\left|U_{\nu 33}\right|^{2}
$$

Since we know that the only solution under the conditions $|a|^{2}+|b|^{2}+|c|^{2}=1$ and $|a|^{2}|b|^{2}|c|^{2} \simeq 1 / 27$ is $|a|^{2} \simeq|b|^{2} \simeq|c|^{2} \simeq 1 / 3$, the relations (25) yield

$$
\left|U_{\nu 13}\right|^{2} \simeq \frac{1}{3}, \quad\left|U_{\nu 23}\right|^{2} \simeq \frac{1}{3},\left|U_{\nu 33}\right|^{2} \simeq \frac{1}{3}
$$

which give

$$
\sin ^{2} 2 \theta_{a t m}=4\left|U_{\nu 23}\right|^{2}\left|U_{\nu 33}\right|^{2} \simeq \frac{4}{9}
$$

The value (28) is too small to explain the observed value [7] $\sin ^{2} 2 \theta_{\text {atm }} \simeq 1.0$, so that the case with $|q|^{2} \simeq 1 / 27$ is ruled out. 
Next, we investigate the case with $|q|^{2} \simeq 0$. By putting

$$
x_{1}=-\varepsilon_{1}, \quad x_{2}=\varepsilon_{2}, \quad x_{3}=1-\varepsilon_{3},
$$

and by putting (29) into the equation (19), we can obtain

$$
\varepsilon_{1} \simeq 2|q|(1-|q|), \quad \varepsilon_{2} \simeq 2|q|(1+|q|), \quad \varepsilon_{3} \simeq 4|q|^{2} .
$$

so that we obtain

$$
\begin{gathered}
\Delta m_{12}^{2} \simeq 4|q| m_{0}^{2}, \quad \Delta m_{23}^{2} \simeq\left(1-2|q|^{2}\right) m_{0}^{2}, \\
\frac{\Delta m_{12}^{2}}{\Delta m_{23}^{2}} \simeq 4|q| .
\end{gathered}
$$

On the other hand, form the relation (24), we obtain

$$
\left(H_{1} / m_{0}^{2}\right)_{i i} \simeq\left|U_{\nu i 3}\right|^{2}-2|q|\left(\left|U_{\nu i 1}\right|^{2}-\left|U_{\nu i 2}\right|^{2}\right)+2|q|^{2}\left(1-3\left|U_{\nu i 3}\right|^{2}\right),
$$

so that we obtain

$$
\left(H_{1} / m_{0}^{2}\right)_{22}=|c|^{2} \simeq\left|U_{\nu 23}\right|^{2}, \quad\left(H_{1} / m_{0}^{2}\right)_{33}=|a|^{2} \simeq\left|U_{\nu 33}\right|^{2},
$$

and

$$
\sin ^{2} 2 \theta_{\text {atm }} \simeq 4|a|^{2}|c|^{2} .
$$

Generally, the only solution of the equation $x y \simeq 1 / 4$ for the positive numbers $x$ and $y$ under the condition $x+y<1$ is $x \simeq y \simeq 1 / 2$. Therefore, the solution of the equation $\sin ^{2} 2 \theta_{\text {atm }}=4\left|U_{\nu 23}\right|^{2}\left|U_{\nu 33}\right|^{2} \simeq 1$ under the condition $\left|U_{\nu 23}\right|^{2}+\left|U_{\nu 33}\right|^{2}=1-\left|U_{\nu 13}\right|^{2}<1$ is

$$
\left|U_{\nu 23}\right|^{2} \simeq\left|U_{\nu 33}\right|^{2} \simeq \frac{1}{2},\left|U_{\nu 13}\right|^{2} \simeq 0
$$

and also the solution of the equation $\sin ^{2} 2 \theta_{\text {atm }}=4|a|^{2}|c|^{2} \simeq 1$ under the condition $|a|^{2}+|c|^{2}=1-|b|^{2}<1$ is

$$
|a|^{2} \simeq|c|^{2} \simeq \frac{1}{2}, \quad|b|^{2} \simeq 0
$$

The result (37) means

$$
|q|^{2} \simeq \frac{1}{4}|b|^{2} .
$$

The $(1,1)$ component of the equation (33) gives

$$
|b|^{2} \simeq\left|U_{\nu 13}\right|^{2}-|b|\left(\left|U_{\nu 11}\right|^{2}-\left|U_{\nu 12}\right|^{2}\right)+\frac{1}{2}|b|^{2}\left(1-3\left|U_{\nu 13}\right|^{2}\right) .
$$


When we put

$$
\left|U_{\nu 11}\right|=\sqrt{1-\left|U_{\nu 13}\right|^{2}} \cos \theta, \quad\left|U_{\nu 12}\right|=\sqrt{1-\left|U_{\nu 13}\right|^{2}} \sin \theta
$$

we obtain

$$
\sin ^{2} 2 \theta_{\text {solar }} \simeq \sin ^{2} 2 \theta \simeq 1-\frac{1}{4}|b|^{2}\left(1-2 \frac{\left|U_{\nu 13}\right|^{2}}{|b|^{2}}\right)^{2}
$$

where

$$
|b| \simeq \frac{1}{2} \frac{\Delta m_{\text {solar }}^{2}}{\Delta m_{\text {atm }}^{2}}
$$

A model which gives $\left|U_{\nu 13}\right|^{2}=0$ cannot obviously give a sizable deviation from $\sin ^{2} 2 \theta_{\text {solar }}=$ 1. However, if $\left|U_{\nu 13}\right|^{2} \sim|b|$, then the value of $\sin ^{2} 2 \theta_{\text {solar }}$ is sensitive to the value of $\left|U_{\nu 13}\right|^{2}$. Therefore, we must estimate the value of $\left|U_{\nu 13}\right|^{2}$ carefully.

We use the relations

$$
\sum_{k=1}^{3}\left(H_{1} / m_{0}^{2}\right)_{i k} U_{\nu k j}=U_{\nu i j} x_{j}
$$

For $j=3$, we obtain

$$
\begin{gathered}
|b|^{2} U_{\nu 13}-c^{*} b U_{\nu 23}-a^{*} b U_{\nu 33}=U_{\nu 13} x_{3}, \\
-b^{*} c U_{\nu 13}+|c|^{2} b U_{\nu 23}-a^{*} c U_{\nu 33}=U_{\nu 23} x_{3}, \\
-b^{*} a U_{\nu 13}-c^{*} a U_{\nu 23}+|a|^{2} U_{\nu 33}=U_{\nu 33} x_{3} .
\end{gathered}
$$

By eliminating $U_{\nu 23}$, we obtain the relation without any approximation

$$
U_{\nu 13}=\frac{-2\left(x_{3}-1+|b|^{2}\right) b a^{*} U_{\nu 33}}{\left(|a|^{2}-|c|^{2}\right)|b|^{2}+\left(x_{3}-|b|^{2}\right)\left(x_{3}-1+|b|^{2}\right)} .
$$

If we use the approximate expression $x_{3} \simeq 1-4|q|^{2} \simeq 1-|b|^{2}$, the factor $\left(x_{3}-1+|b|^{2}\right)$ becomes vanishing. Therefore, in order to estimate the factor $\left(x_{3}-1+|b|^{2}\right)$ more precisely, we use the following expression of $x_{3}$ to the order of $|q|^{4}$,

$$
x_{3} \simeq 1-4|q|^{2}\left(1+8|q|^{2}\right)
$$

Then, we can show

$$
x_{3}-1+|b|^{2} \simeq|b|^{2}\left[\left(|a|^{2}-|c|^{2}\right)^{2}+7|b|^{4}\right] .
$$


Since we know that $|b|^{2}$ is very small value, i.e., $|b|^{2} \simeq(1 / 4)\left(\Delta m_{\text {solar }}^{2} / \Delta m_{\text {atm }}^{2}\right)^{2}$, we investigate only the case $\left(|a|^{2}-|c|^{2}\right)^{2} \geq|q|^{4}$. Then, from Eq. (47), we can obtain

$$
U_{\nu 13} \simeq-2\left(|a|^{2}-|c|^{2}\right) b a^{*} U_{\nu 33}
$$

i.e.,

$$
\left|U_{\nu 13}\right|^{2} \simeq\left(|a|^{2}-|c|^{2}\right)^{2}|b|^{2} .
$$

On the other hand, we can show that the quantities $\left(\Delta m_{12}^{2} / \Delta m_{23}^{2}\right)^{2}$ and $\sin ^{2} 2 \theta_{\text {atm }}=$ $4\left|U_{\nu 23}\right|^{2}\left|U_{\nu 33}\right|^{2}$ are insensitive to the parameter $\left(|a|^{2}-|c|^{2}\right)$. Therefore, from Eqs.(47) and (51), we can obtain the following parameter-independent relation

$$
\sin ^{2} 2 \theta_{\text {solar }} \simeq 1-\frac{1}{4}\left[1-2\left(|a|^{2}-|c|^{2}\right)^{2}\right]^{2}|b|^{2} \geq 1-\frac{1}{16}\left(\frac{\Delta m_{\text {solar }}^{2}}{\Delta m_{\text {atm }}^{2}}\right)^{2}
$$

where we have used $\left[1-2\left(|a|^{2}-|c|^{2}\right)^{2}\right]^{2} \leq 1$.

The constraint (52) cannot be loosened even if we consider the renormalization group equation (RGE) effects. The mass matrix form (1) is given by the radiative diagrams at the low energy scale, where the charged lepton mass matrix is given by the diagonal form. Although the coupling constants $f_{i j}$ given in Eq. (2) are affected by the RGE, since our conclusion (52) is independent of the explicit values of the parameters $a, b$ and $c$ in Eq. (1), the conclusion (52) cannot be loosen even by taking RGE effects into consideration.

However, we must note that the mass matrix form (1) based on only the one-loop radiative mass diagrams. When we take two-loop diagrams into consideration, as pointed out by Chang and Zee [12], non-vanishing contributions appear in the diagonal elements of $M_{\nu}$. For the case which gives $\sin ^{2} 2 \theta_{\text {atom }} \simeq 1$, the relations (37) are required, so that the relations $\left|f_{e \mu}\right| m_{\mu}^{2} \simeq\left|f_{e \tau}\right| m_{\tau}^{2} \gg\left|f_{\mu \tau}\right| m_{\tau}^{2}$ are required. Then, as discussed in Ref. [12], we can estimate

$$
\left|M_{\nu 12}\right| \simeq\left|M_{\nu 13}\right| \gg\left|M_{\nu 23}\right|>\left|M_{\nu 11}\right| \simeq\left|M_{\nu 22}\right| \gg\left|M_{\nu 33}\right|,
$$

where $\left|M_{\nu i j}\right| \propto f_{i j}\left(m_{i}^{2}-m_{j}^{2}\right)$ and $\left|M_{\nu i i}\right| \propto\left|f_{12}\right|\left|f_{23}\right|\left|f_{31}\right|\left(m_{j}^{2}-m_{k}^{2}\right)(j \neq i \neq k)$. We interest in a value of the ratio $\left|M_{\nu 11} / M_{\nu 23}\right|$. If the ratio is negligibly small, the result (52) will be still valid, but if the ratio is sizable, then the result (52) will be valid no more. According to Ref. [12], we estimate $\left|M_{\nu 11} / M_{\nu 23}\right|$ as

$$
\left|\frac{M_{\nu 11}}{M_{\nu 23}}\right| \sim \frac{\left|f_{e \mu}\right|\left|f_{\mu \tau}\right|\left|f_{\tau e}\right|}{16 \pi^{2}\left|f_{\mu \tau}\right|} \simeq \frac{\left|f_{e \mu}\right|^{2}}{16 \pi^{2}}\left(\frac{m_{\mu}}{m_{\tau}}\right)^{2}<10^{-5} .
$$

Therefore, we conclude that the severe constraint (52) is still valid even if we take two-loop diagrams into consideration. 
However, note that if the mass matrix (1) is not due to the Zee mechanism, but due to a seesaw mechanism, $M_{\nu} \simeq-m_{L} M_{R}^{-1} m_{L}^{T}$, the form of $M_{\nu}$ will be changed by the RGE effects.

Therefore, we can conclude that when we require $\Delta m_{\text {solar }}^{2} / \Delta m_{\text {atm }}^{2} \ll 1$ for the Zee model, although we can give $\sin ^{2} 2 \theta_{\text {atm }} \simeq 1$, but, at the same time, the value of $\sin ^{2} 2 \theta_{\text {solar }}$ must also be highly close to one. On the other hand, in contrast to the theoretical bound (52), the best fit value of $\sin ^{2} 2 \theta_{\text {solar }}$ is

$$
\sin ^{2} 2 \theta_{\text {solar }} \simeq 0.66
$$

for the MSW LMA solution [9]. The prediction $\sin ^{2} 2 \theta_{\text {solar }} \simeq 1.0$ is in poor agreement with the observed data (in the outside of the region 99\% C.L.). Of course, the value (55) is a best-fit value, and it does not mean that the Zee model is ruled out. However, if the data in future exclude the value $\sin ^{2} 2 \theta_{\text {solar }} \simeq 1.0$ completely, we will be forced to abandon the Zee model, at least, for the MSW LMA solution. At present, if we still adhere to the Zee model, the only solution which we should take is the Just $\mathrm{So}^{2}$ solution [13] with $\sin ^{2} 2 \theta_{\text {solar }} \simeq 1.0$. However, the Just $\mathrm{So}^{2}$ solution does not always the best one of the possible candidates (the best fit solutions) at present (for example, the MSW LMA solution gives $\chi_{\min }^{2}=29.0$, while the Just $\mathrm{So}^{2}$ solution $\chi_{\min }^{2}=36.1$ [9]).

In conclusion, we have investigated the Zee neutrino mass matrix (1) parameterindependently. When we require that the value $\Delta m_{\text {solar }}^{2} / \Delta m_{\text {atm }}^{2}=\Delta m_{12}^{2} / \Delta m_{23}^{2}$ should be very small, the possible solutions are only two cases with $|q|^{2} \equiv|a|^{2}|b|^{2}|c|^{2} \simeq 1 / 27$ and $|q|^{2} \simeq 0$ where $|a|^{2}+|b|^{2}+|c|^{2}=1$. The case with $|q|^{2} \simeq 1 / 27$ leads to $\sin ^{2} 2 \theta_{\text {atm }} \simeq 4 / 9$, so that the case is ruled out. The case with $|q|^{2} \simeq 0$ leads not only to $\sin ^{2} 2 \theta_{\text {atm }} \simeq 1$, but also to $\sin ^{2} 2 \theta_{\text {solar }} \geq 1-(1 / 16)\left(\Delta m_{\text {solar }}^{2} / \Delta m_{\text {atm }}^{2}\right)^{2}$. The prediction $\sin ^{2} 2 \theta_{\text {solar }} \simeq 1.0$ is in poor agreement with the observed data. However, in spite of such a problem, the Zee model is still attractive to us, because the model can naturally lead to a nearly bimaximal mixing. Therefore, we would like to expect that the problem will be overcome by some future modification of the original Zee model. For examples, the following attempts will be promising: introducing a new doubly charged scalar $k^{++}$in order to obtain sizable two-loop contributions [14], and introducing right-handed neutrinos in order to additional mass terms, and embedding the original Zee model into an $R$-parity violating SUSY model [15] and into an $R$-parity conserving SUSY model [16], and so on.

\section{Acknowledgments}

The author would like to thank A. Ghosal and H. Fusaoka for their helpful discussions and comments, and Midori Kobayasi for informing a mathematical theorem in uniqueness of the solutions. He also thank S. T. Petcov for informative discussion and valuable comments. Furthermore, he is also grateful to M. Yasue for valuable discussion 
on the two-loop effects in the Zee model, to A. Zee for his quick response to an inquiry about the two-loop effects, and to M. Tanimoto and J. Sato for helpful discussions on SUSY versions of the Zee model.

\section{References}

[1] A. Zee, Phys. Lett. 93B, 389 (1980); 161B, 141 (1985); L. Wolfenstein, Nucl. Phys. B175, 93 (1980).

[2] S. T. Petcov, Phys. Lett. 115B, 401 (1982).

[3] A. Yu. Smirnov and M. Tanimoto, Phys. Rev. D55, 1665 (1997).

[4] C. Jarlskog, M. Matsuda, S. Skadhauge, M. Tanimoto, Phys. Lett. B449, 240 (1999).

[5] A. Ghosal, Phys. Rev. D62, 092001 (2000).

[6] Y. Koide and A. Ghosal, Phys. Rev. D63, 037301 (2001).

[7] Y. Fukuda et al., Phys. Lett. B335, 237 (1994); Super-Kamiokande collaboration, Y. Fukuda, et. al., Phys. Rev. Lett. 81, 1562 (1998); H. Sobel, Talk presented at Neutrino 2000, Sudbury, Canada, June 2000 (http://nu2000.sno.laurentian.ca/).

[8] M. Gonzalez-Garcia, Talk presented at Neutrino 2000, Sudbury, Canada, June 2000 (http://nu2000.sno.laurentian.ca/).

[9] J. N. Bahcall, P. I. Krastev and A. Yu. Smirnov, hep-ph/0103179.

[10] P. H. Frampton and S. L. Glashow, Phys. Lett. B461, 95 (1999).

[11] Z. Maki, M. Nakagawa and S. Sakata, Prog. Theor. Phys. 28, 870 (1962).

[12] D. Chang and A. Zee, Phys. Rev. D61, 071303(R) (2000).

[13] R. S. Raghavan, Solar neutrinos - form puzzle to paradox, Science 267, 45 (1995).

[14] A. Zee, Nucl. Phys. 264B, 99 (1986); K. S. Babu, Phys. Lett. B203, 132 (1988); D. Chang, W.-Y. Keung and P. B. Pal, Phys. Rev. Lett. 61, 2420 (1988). For a recent work, see for example, T. Kitabayashi and M. Yasue, Phys. Lett. B490, 236 (2000).

[15] M. Drees, S. Pakvasa, X. Tata and T. ter Veldhuis, Phys. Rev. D57, 5335 (1998); G. Bhattacharyya, H. V. Klapdor-Kleingrothaus and H. Pas, Phys. Lett. B463, 77 (1999); K. Cheung and O. C. W. Kong, Phys. Rev. D61, 113012 (2000).

[16] For instance, N. Haba, M. Matsuda and M. Tanimoto, Phys. Lett. B478, 351 (1999). 